\title{
Is Cytomegalovirus a Neglected Pathogen in the Field of Immunobiology?
}

Rodney $\mathbf{P}$ Jones

Department of Healthcare Analysis and Forecasting, Royal Berkshire Hospital, UK

*Corresponding author: Rodney P Jones, Department of Healthcare Analysis and Forecasting, Royal Berkshire Hospital, UK, E-mail: hcaf_rod@yahoo.co.uk

Received date: November 29, 2018; Accepted date: December 27, 2018; Published date: January 04, 2019

Copyright: @2018 Jones RP. This is an open-access article distributed under the terms of the Creative Commons Attribution License, which permits unrestricted use, distribution, and reproduction in any medium, provided the original author and source are credited.

\begin{abstract}
Around the world deaths are behaving in a unique way which was previously thought to be impossible, namely, they are showing on/off switching. In switch-on they suddenly jump to a new higher level, stay high for around 12months, and then switch-off back to the baseline trajectory. This behaviour originates at small (neighbourhood) area level and aggregates to give regional and national events whose magnitude depends on the degree of small-area synchrony. This mini-review explores the possibility that the common immune-modifying virus cytomegalovirus may be involved in these events
\end{abstract}

Keywords: Deaths; Medical admissions; Sickness absence; Infectious spread; On/off switching; Cytomegalovirus

\section{Introduction}

Evidence has recently emerged that deaths in all Western countries are behaving in a way previously thought to be impossible, in that they are showing on/off switching [1]. At switch-on deaths suddenly jump to a higher level, they stay high for around 12 months, at which point switch-off occurs and deaths revert to the lower baseline position. Deaths then stay at baseline until the next switch-on event.

Clearly something must trigger switch-on. Interestingly, medical admissions to hospital [2], hospital staff sickness absence [3] and inhospital deaths all show the same behaviour [4]. This behaviour arises exclusively at small-area (neighbourhood) level indicating the possible transmission of a pathogen along social networks. Males and females behave as separate compartments and certain social groups appear more at risk of infection [5,6]. Persons with Alzheimer's and dementia show the highest mortality during these events [7], followed by those with lung infections [8].

\section{The Link between Deaths and Medical Admissions}

The link between deaths and medical admissions occurs via the nearness to death effect, where around half of a person's lifetime hospital admissions and bed occupancy is compressed into the last year of life, irrespective of the age at death [9]. The intrinsic high volatility in deaths therefore drives the marginal changes in admissions and bed occupancy, which explains why forecasting hospital demand has been such a problematic issue. The last year of life witnesses a rapid decline in cognitive and functional ability and increasing dependence on carers for assistance $[10,11]$.

Hence any agent capable of tipping a susceptible fraction of the population into their last year of life would create the curious 12month duration for switch-on observed in deaths and medical admissions, although deaths somewhat understandably lag admissions, i.e. acute illness precedes ultimate decease. This explains the relationship with staff sickness absence via exposure to patients.

\section{Which Pathogen(s) could be Involved?}

Hence, we have a seemingly transmissible agent capable of triggering illness in otherwise healthy hospital staff and of increasing medical admissions and deaths, mainly in the elderly.

Indeed, the agent must have a subtle effect in order to avoid detection. Bacteria would almost certainly be detected, leaving a virus as the most likely cause. Influenza has been rejected as a possible cause since persistent infection would be required to create a 12-month duration switch-on effect.

Clearly, any persistent virus could qualify, and most of the herpes viruses are widely prevalent around the world and have periods of reactivation.

However, based upon the range of medical conditions (including certain cancers) associated with each event, cytomegalovirus (CMV) has been suggested as a possible candidate. The following are relevant [12-17].

1. Despite assurances in medical textbooks, CMV is increasingly being diagnosed as a cause for a wide variety of acute illnesses in the seemingly immunocompetent patient

2. High levels of CMV IgG with inflammation are associated with substantially higher population mortality - a necessary condition for the observed higher deaths

3. CMV has a largest genome of all common viruses which is dedicated to diverse aspects of immune manipulation

4. $\mathrm{CMV}$ is now recognized as both oncomodulatory and oncogenic

5. CMV exerts aspects of immune modulation whilst seemingly 'dormant'

6. Infection and reinfection with multiple CMV strains are common

7. CMV is known to cause localized mini-outbreaks

8. Infants and children shed CMV for many months and can therefore act as super-spreaders

9. $\mathrm{CMV}$ is central in the expression of the infectious burden, i.e. the action of multiple acquired pathogens 
10. English GP’s have a $0 \%$ success rate at diagnosing patients with a CMV infection

While the above list does not conclusively prove that CMV is the causative agent, it suggests that it is likely to be a strong contender. A recent study using CMV infection in rhesus macaques has demonstrated that subclinical infection simultaneously alters host immunity, the gut microbiota and vaccine responses [18]. Such studies suggest that $\mathrm{CMV}$ is certainly capable of the diverse changes required to lead to switch-on in a susceptible fraction of the population.

Preliminary calculations indicate that around $20 \%$ of the population may be sensitive to the effects of the agent [8] and this suggests that genetic factors may also be involved. Indeed, certain strains of CMV are more prevalent in some countries than others [12-17].

Clearly no pathogen acts in isolation, and with over 1,400 known human pathogens [19], it would be surprising if the actual agent was not acting in concert with other persistent pathogens (the infectious burden), and CMV is a common denominator in many studies investigating the role of the infectious burden upon specific diseases [12-17].

If $\mathrm{CMV}$ is the causative agent, dedicated immune and genetic studies will need to be involved to elucidate how subclinical infection can alter the course of a variety of diseases. Even if CMV is not involved we need explanations as to how population health can show on/off switching, and such investigations will almost certainly involve new understanding relating to wider immune system balance and switching between alternative steady states [20].

\section{References}

1. Jones R (2017) Outbreaks of a presumed infectious pathogen creating on/off switching in deaths. SDRP J Infect Dis Treat Therapy 1: 1-6.

2. Jones $\mathrm{R}$ (2015) Small area spread and step-like changes in emergency medical admissions in response to an apparently new type of infectious event. Fractal Geometry and Nonlinear Anal in Med and Biol 1: 42-54.

3. Jones R (2016) Unusual trends in NHS staff sickness absence. Brit J Health Manage22 : 239-240.

4. Jones R (2018) Unexpected trends in hospital standardized mortality indicate a novel cause. Eur J Internal Med 52: 9-11.

5. Jones R (2016) A regular series of unexpected and large increases in tota deaths (all-cause mortality) for male and female residents of mid super output areas (MSOA) in England and Wales: How high-level analysis can miss the contribution from complex small-area spatial spread of a presumed infectious agent. Fractal Geometry and Nonlinear Anal in Med and Biol 2: 1-13.
6. Jones R (2017) Role of social group and gender in outbreaks of a novel agent leading to increased deaths, with insights into higher international deaths in 2015. Fractal Geometry and Nonlinear Anal in Med and Biol 3: $1-14$.

7. Jones R, Goldeck D (2014) Unexpected and unexplained increase in death due to neurological disorders in 2012 in England and Wales: Is cytomegalovirus implicated? .Med Hypotheses 83: 25-31.

8. Jones R (2014) A study of an unexplained and large increase in respiratory deaths in England and Wales: Is the pattern of diagnoses consistent with the potential involvement of Cytomegalovirus? Brit J Med Medical Res 4: 5179-5192.

9. Hanlon P, Walsh D, Whyte BW, Scott SN, Lightbody P et al. (1998) Hospital use by an ageing cohort: an investigation into the association between biological, behavoural and social risk markers and subsequent hospital utilization. J Public Health Med 20: 467-476.

10. Rabbitt P, Lunn M, Wong D (2008) Death, dropout, and longitudinal measurements of cognitive change in old age. J Gerontol Psych Sci 63: 271-278.

11. Kalbarczyk-Steclik M, Nicinska A (2015) The last and the previous year of life in Europe: A comparative analysis of care received and daily living limitations. J Aging Gerontol 3: 1-7.

12. Jones R (2013) Could cytomegalovirus be causing widespread outbreaks of chronic poor health? In Hypotheses in Clinical Medicine New York: Nova Science Publishers Inc. 37-79

13. Jones R (2013) Widespread outbreaks of a subtle condition leading to hospitalization and death. Epidemiology: Open access 4: 137.

14. Jones R (2015) Roles for cytomegalovirus in infection, inflammation and autoimmunity. In Infection and Autoimmunity, 2nd Edition, Eds: N Rose, et al. Elsevier: Amsterdam. Chapter 18, 319-357.

15. Jones R (2015) An unexpected increase in adult appendicitis in England (2000/01 to 2012/13): Could cytomegalovirus (CMV) be a risk factor? Brit J Med Medical Res 5: 579-603.

16. Jones R (2016) Is cytomegalovirus involved in recurring periods of higher than expected death and medical admissions, occurring as clustered outbreaks in the northern and southern hemispheres? Brit J Med Medical Res 11: 1-31.

17. Jones $\mathrm{R}$ (2017) International outbreaks of a novel type of infectious immune impairment: A call to action. Achievements of Biology and Medicine 29: 75-81.

18. Rocha C, Hirao L, Weber M, Mendez-Lagares G, Chang W, et al. (2018) Subclinical cytomegalovirus infection is associated with altered host immunity, gut microbiota, and vaccine responses. J Virol 92: 167-218.

19. Woolhouse M, Gowtage-Sequeria S (2005) Host range and emerging and reemerging pathogens. Emerg Infect Dis 11: 1842-1847.

20. Craddock T, Fritsch P, Rice M, Miller DB, Fletcher MA ,et al. (2014) A role for homeostatic drive in the perpetuation of complex chronic illness: Gulf war illness and chronic fatigue syndrome. PLoS One 9: 84839. 